\title{
Inverted Follicular Keratosis
}

National Cancer Institute

\section{Source}

National Cancer Institute. Inverted Follicular Keratosis. NCI Thesaurus. Code C9007.

Seborrheic keratosis that arises from follicular structures in the skin. It presents as a solitary nodule in the skin and is characterized by the presence of prominent squamous eddies. 